\title{
Meiotic MCM proteins promote and inhibit crossovers during meiotic recombination
}

4 *Curriculum in Genetics and Molecular Biology, University of North Carolina, Chapel Hill,

$5 \quad$ North Carolina 27599

$6{ }^{\dagger}$ Department of Biology, Winthrop University, 701 Oakland Avenue, Rock Hill, SC 29733

$7{ }^{\ddagger}$ Department of Biology, University of North Carolina, Chapel Hill, North Carolina 27599

$8{ }^{\S}$ Integrative Program in Biological and Genome Sciences, University of North Carolina, Chapel

$9 \quad$ Hill, North Carolina 27599

$10 \quad{ }^{* *}$ Corresponding author

11

12 Running title: Genetic analysis of Drosophila mei-MCMs

13

14 Five key words or phrases: Drosophila, meiotic recombination, meiosis, crossover, mei-MCM

16 Corresponding author: CB \#3280, 303 Fordham Hall, Curriculum of Genetics and Molecular

17 Biology, University of North Carolina, Chapel Hill, NC 27599-3280.

18 E-mail: hatkevic@email.unc.edu 
Abstract

Crossover formation as a result of meiotic recombination is vital for proper segregation

21 of homologous chromosomes at the end of meiosis I. In many organisms, crossovers are

22 generated through two crossover pathways: Class I and Class II. To ensure accurate crossover

23 formation, meiosis-specific protein complexes regulate the degree in which each pathway is

24 used. One such complex is the mei-MCM complex, which contains MCM (mini-chromosome

25 maintenance) and MCM-like proteins REC (ortholog of Mcm8), MEI-217, and MEI-218,

26 collectively called the mei-MCM complex. The mei-MCM complex genetically promotes Class I

27 crossovers and inhibits Class II crossovers in Drosophila, but it is unclear how individual mei-

$28 \mathrm{MCM}$ proteins contribute to crossover regulation. In this study, we perform genetic analyses to

29 understand how specific regions and motifs of mei-MCM proteins contribute to Class I and II

30 crossover formation and distribution. Our analyses show that the long, disordered N-terminus of

31 MEI-218 is dispensable for crossover formation, and that mutations that disrupt REC's Walker A

32 and B motifs differentially affect Class I and Class II crossover formation. In Rec Walker A

33 mutants, Class I crossovers exhibit no change, but Class II crossovers are increased. However, in

34 rec Walker B mutants, Class I crossovers are severely impaired, and Class II crossovers are

35 increased. These results suggest that REC may form multiple complexes that exhibit differential

36 REC-dependent ATP binding and hydrolyzing requirements. These results provide genetic

37 insight into the mechanisms through which mei-MCM proteins promote Class I crossovers and

38 inhibit Class II crossovers. 


\section{Introduction}

To reestablish the diploid genome upon sexual fertilization, the genome of progenitor

41 germ cells must be successfully reduced by half through meiosis. Accurate reduction of the

42 genome at the end of meiosis I requires crossover formation between homologous chromosomes

43 during meiotic recombination. Meiotic recombination is initiated by the formation of multiple

44 double-strand breaks (DSBs); the majority of meiotic DSBs are repaired as noncrossovers, while

45 a selected subset are repaired as crossovers between homologs (reviewed in Lake and Hawley 46 2012).

47 Two distinct types of meiotic crossovers have been described: Class I and Class II. First defined in budding yeast (de Los Santos et al. 2003), Class I and Class II crossovers exist in most

49 sexually reproducing organisms, but the relative proportions of each crossover type vary among 50 organisms (Hollingsworth and Brill 2004). In Drosophila, most - if not all - crossovers are

51 generated through the Class I pathway (Hatkevich et al. 2017), as shown through their depen52 dence on the putative catalytic unit of the Class I meiotic resolvase MEI-9 (Sekelsky et al. 1995;

53 Yildiz et al. 2002) and their display of crossover interference (Hatkevich et al. 2017). Most 54 crossovers in Drosophila are also dependent upon a group of MCM- or MCM-like proteins, 55 called the mei-MCM complex (Baker and Carpenter 1972; Grell 1978; Liu et al. 2000; Kohl et 56 al. 2012).

The mei-MCM complex consists of REC (the Drosophila ortholog of MCM8), MEI-217,

58 and MEI-218. REC appears to be a bona fide MCM protein, based on conservation of both the

59 N-terminal MCM domain and the C-terminal AAA+ ATPase domain, which includes Walker A 60 and B boxes that bind and hydrolyze ATP (Figure 1A). In contrast, MEI-217 and MEI-218 are 61 highly divergent MCM-like proteins, and together resemble one full MCM protein. MEI-217 is 
62 structurally similar to the MCM N-terminal domain, though this similarity is not detected in

63 BLAST or conserved domain searches (Kohl et al. 2012). The carboxy-terminus of MEI-218 has

64 a domain related to the AAA+ ATPase domain, but key residues are not conserved, including the

65 Walker A and B motifs that are critical for binding and hydrolyzing ATP, respectively (Iyer et al.

66 2004) (Figure 1B). Because key residues in the Walker A and B motifs are not conversed,

67 MEI-218 may not exhibit ATPase activity or it may exhibit partial function. In addition,

68 MEI-218 has a long N-terminal extension that is poorly conserved and predicted to be

69 disordered. The function of this region is unknown, but gene swap studies suggest that it may

70 contribute to differences in the recombination landscape among Drosophila species (Brand et al.

71 2018). For further analysis and details regarding the evolution of the mei-MCM complex, see

72 Supplemental Figures S1-S3.

73 While most crossovers are generated through the Class I pathway in wild-type

74 Drosophila and are mei-MCM dependent, mutants that lack the Bloom syndrome helicase (Blm)

75 generate only Class II crossovers based on their independence of MEI-9 and lack of patterning

76 (e.g., interference) that is associated with Class I crossovers (Hatkevich et al. 2017). Blm is an

77 ATP-dependent 3'-5' helicase that exhibits vital anti-crossover functions in both meiotic and

78 somatic DSB repair (reviewed in Hatkevich and Sekelsky 2017). Interestingly, mutations in mei-

$79 M C M$ and Blm genes genetically interact. In Blm mutants, crossovers are reduced by $30 \%$ but in

80 a Blm rec double mutant, crossovers are significantly increased compared to wild-type (Kohl et

81 al. 2012). This suggests that the mei-MCMs may function to inhibit crossovers within the Class

82 II pathway, in addition to their role promoting crossovers in the Class I pathway.

83 While the mei-MCMs function as a complex, little is known about how individual

84 mei-MCMs contribute to Class I and II crossover regulation. Here, we investigate specific 
85 features of MEI-218 and REC to understand better how these proteins contribute to meiotic

86 recombination. We find that the N-terminus of MEI-218 is dispensable for crossover formation

87 and general crossover distribution. By mutating key residues in REC's Walker A and B motifs

88 (rec ${ }^{K A}$ and $\mathrm{rec}^{D A}$, respectively), we found that $\operatorname{rec}^{K A}$ mutants exhibit no Class I crossover defect,

89 while Class II crossovers are significantly increased. Surprisingly, rec $^{D A}$ mutants exhibit a severe

90 decrease in Class I crossovers and a significant increase in Class II crossovers. Our results

91 suggest that the mei-MCMs function in multiple roles and may complex in a variety of

92 configurations to properly regulate crossover formation.

\section{Materials and Methods}

94 Drosophila stocks. Flies were maintained on standard medium at $25^{\circ} \mathrm{C}$. Some mutant alleles

95 have been previously described, including mei-9 $9^{a}$ (Baker and Carpenter 1972; Y1ldiz et al. 2004),

$96 m^{m i-218^{l}}$ and $m e i-218^{6}$ (Baker and Carpenter 1972; Mckim et al. 1996), Blm ${ }^{N 1}$ and Blm ${ }^{D 2}$

97 (Mcvey et al. 2007), rec $^{1}$ and rec $^{2}$ (Grell 1978; Matsubayashi and Yamamoto 2003; Blanton et

98 al. 2005). The maternal-effect lethality in $B l m^{N 1} / B l m^{D 2}$ mutants was overcome by the

99 UAS::GAL4 rescue system previously described (Kohl et al. 2012).

100 Generating mei-218 transgenic alleles. The transgenes for mei-218 $\Delta^{N}$ and $m e i-218^{F L}$ were

101 constructed by cloning cDNA for mei-218 into $P\{$ attBUASpW $\}$ (AddGene). Full-length mei-218

102 included codons 1-1186; the mei-218 $\Delta^{N}$ transgene included codons 527-1186. Transgenics were

103 made by integrating into a phiC31 landing site in 2A on the $X$ chromosome.

104 Generating rec $^{\mathrm{KA}}$ and rec $^{\mathrm{DA}}$ mutants. Annealed oligonucleotides were inserted into BbsI105 digested pU6-BbsI-chiRNA plasmid $\quad$ rec $^{K A}$ : 106 CTTCGCCGAGAAGGGATAGTAAAC; $r e c^{D A}$ : CTTCGTTGCAGTGCCTACAATCAG. 
107 Resulting plasmids were co-injected with repair template plasmid, consisting of synthesized 108 gBlocks (IDT DNA) cloned into pBlueScript plasmid (sequences available on request). Injected

109 larvae were raised to adulthood, and their male progeny were crossed to TM3/TM6B females

110 (Bloomington Stock Center) to generate stocks, after which DNA was extracted for screening

111 through PCR and restriction digest.

112 Nondisjunction assay. X-chromosome nondisjunction (NDJ) was assayed by mating virgin

113 females to $y c v v f / T(1: Y) B^{S}$ males. Each cross was set up as a single experiment with 20-50

114 separate vials. The progeny of each vial were counted separately. Viable nondisjunction progeny

115 are $X X Y$ females with Bar eyes and $X O$ males with Bar $^{+}$eyes and the phenotypes from $y c v v f$

116 chromosome. Total (adjusted) represents the total with inviable exceptional progeny accounted

117 for $(X X X$ and $Y O)$. NDJ rates and statistical comparisons were done as in Zeng et al. 2010.

118 Crossover distribution assay. Crossover distribution on chromosome 2L was scored by crossing

119 virgin net $d p p^{d-h o} d p$ b pr cn / + female flies with mutant background of interest to net $d p p^{d-h o} d p$

$120 \quad b \mathrm{pr}$ cn homozygous males. Each cross was set up as a single experiment with at least 25

121 separate vials scored. The first set of vials was flipped after three days of mating into vials of a

122 new batch, although these were counted as one experiment. Batch effects for recombination

123 assays have not been observed in repeated studies for multiple genotypes used in this study

124 (Figure S4). These include wild-type (unpublished data), Blm (unpublished data), rec (Blanton et 125 al. 2005; Kohl et al. 2012), mei-9 (Sekelsky et al. 1995), and mei-9; rec (Blanton et al. 2005).

126 All progeny were scored for parental and recombinant phenotypes. Crossover numbers in flies

127 are shown as $\mathrm{cM}$ where $\mathrm{cM}=$ (number of crossovers / total number of flies) $* 100$. Chi-squared

128 tests with Bonferroni correction were performed for each interval. For total cM, Fisher's Exact

129 Test was used to compare total crossovers to total number of flies. Crossover distribution is 
130 represented as $\mathrm{cM} / \mathrm{Mb}$ where $\mathrm{Mb}$ is length of the interval without transposable elements (TEs)

131 because crossovers rarely occur within TEs (Miller et al. 2016).

132 Protein structure and alignment. Structural domains of proteins were determined by using

133 PHYRE 2. All of the MCM regions identified correspond to the protein data bank ID \#c2vl6C

134 and the AAA ATPase domains identified correspond to protein data bank ID \#d1g8pa.

135 Alignment of the Walker A and Walker B motifs (Kohl et al. 2012) was done using MEGA 5

136 and aligned with the ClustalW program. Identical and conserved residues are shaded based on

137 groups of amino acids with similar chemical properties.

138 Data availability. All data necessary for confirming the conclusions in this paper are included in

139 this article and in supplemental figures and tables. Drosophila stocks and plasmids described in

140 this study are available upon request. We have uploaded Supplemental Material to Figshare.

141 Figure S1 illustrates distribution of Msh4, Msh5, Mcm8, Mcm9, MEI-217, and MEI-218 in

142 Diptera. Figure S2 illustrates the structure of MEI-217 and MEI-218 in Diptera. Figure S3 shows

143 sequence alignment of MEI-218. Figure S4 compares crossover frequencies in different batches

144 of the same genotype. Figure S5 details the cross scheme of mei-218 transgene experiments.

145 Table S1 includes analysis of genetic interval differences between $W T$ and $m e i-218^{F L}$. Table S1

146 includes analysis of genetic interval differences between mei- $218^{F L}$ and $m e i-218^{\Delta N}$. Table S2

147 includes complete data set for calculating nondisjunction of $W T, \mathrm{rec}^{-} / \mathrm{rec}^{+}$, and $\mathrm{rec}^{D A} /+$. Table S3

148 includes all data sets for meiotic crossovers for all genotypes discussed. 


\section{Results and Discussion}

The N-terminus of MEI-218 is dispensable for crossover formation

MCMDC2 is a distantly-related member of the MCM family of proteins that is unique in that the ATPase domain is predicted to be incapable of binding or hydrolyzing ATP. Orthologs in Dipteran insects are further distinguished by having the N-terminal and ATPase-like domains encoded in separate open reading frames. The two polypeptides, MEI-217 and MEI-218 interact physically, at least in Drosophila melanogaster, presumably reconstituting a single MCM-like protein. MEI-218 is also distinguished by possessing an $\mathrm{N}$-terminal extension of variable length in different species. Drosophila melanogaster MEI-218 can be divided into three distinct regions (Figure 1A): an N-terminal tail (residues 1-500), a central acidic region (residues 500-800) and the C-terminal ATPase-related region (residues 850-1116) (Kohl et al. 2012; Brand et al. 2018). The N-terminal and middle regions are predicted to be disordered (Kohl et al. 2012) and are poorly conserved (Figure S3). Results obtained through gene swap experiments suggest that the N-terminal tail and central region regulate crossover number and distribution within Drosophila species (Brand et al. 2018).

To genetically examine the function of the N-terminus of MEI-218, we compared functions of a transgene that expresses a truncated form of MEI-218 that lacks the N-terminal 526 amino acids $\left(m e i-218^{\Delta^{N}}\right)$ to a matched full-length transgene (mei-218 $8^{F L}$ ) (Figure 2A). Due to the relatively high conservation among Drosophila species, the middle region of mei-218 was retained for this experiment (Figure S3). Using the UAS/GAL4 system (Duffy 2002), we expressed both constructs in mei-218 null mutants using the germline-specific nanos promoter and measured crossovers along five adjacent intervals that span most of $2 \mathrm{~L}$ and part of $2 R$ (Figure S4; for simplicity, we refer to this chromosomal region as 2L.) 
In wild-type females, the genetic length of $2 \mathrm{~L}$ is $45.8 \mathrm{cM}$ (Hatkevich et al. 2017) (Figure

173 2B), whereas mei-218 mutants exhibit a severe decrease in crossovers, with genetic length of

$1742.92 \mathrm{cM}$ (Kohl et al. 2012). Expression of mei-218 $8^{F L}$ in mei-218 mutants (mei-218 ${ }^{F L}$ ) fully

175 rescues the crossover defect, exhibiting a genetic length of $54.1 \mathrm{cM}$. Unexpectedly, expression of

$176 m e i-218^{\Delta^{N}}$ in $m e i-218$ mutants $\left(m e i-218^{\Delta^{N}}\right)$ restored crossing over to the same level as in

177 mei-218; mei-218 ${ }^{F L}(55.9 \mathrm{cM}$; n.s. $p=0.61)$.

178 Brand et al. (2018) expressed Drosophila mauritiana MEI-217 and MEI-218 in

179 Drosophila melanogaster and found that crossovers were increased in proximal and distal

180 regions, resulting in an overall change in crossover distribution. We examined crossover

181 distribution in $m e i-218 ; m e i-218^{F L}$ and $m e i-218$; $m e i-218^{N}$ (Figure 2C). Overall, distributions

182 are similar, with both genotypes exhibiting a strong inhibition of crossovers near the centromere

183 (referred to as the centromere effect; Beadle 1932) and the majority of the crossovers placed in

184 the medial-distal regions (Figure 2C).

185 We conclude that the N-terminal tail of MEI-218 is dispensable for both crossover

186 formation and overall distribution on chromosome 2L. This conclusion is supported by the

187 observation that of 16 sequenced mutations in Drosophila melanogaster mei-218, 14 are

188 nonsense or frameshift, and the only two missense mutations alter residues in the C-terminus

189 (amino acids 845 and 1107) (Collins et al. 2012).

The reasons why the MCM domains have been separated into MEI-217 and MEI-218

191 polypeptides and why MEI-218 has an N-terminal extension are unknown, but this structure has

192 been maintained for more than 250 million years of Dipteran evolution (Supplemental Figures

193 S2). Interestingly, MEI-218 is expressed moderately highly in testes (Thurmond et al. 2018)

194 even though males do not experience meiotic recombination. The predominant or exclusive 
transcript in males does not encode MEI-217 (Thurmond et al. 2018), the seemingly obligate partner for MEI-218 the female meiotic recombination. Males that lack mei-218 are viable,

197 fertile, and do not exhibit elevated nondisjunction (Baker and Carpenter 1972; Mckim et al.

198 1996). For these reasons, we speculate that an unknown function of MEI-218 (independent of

199 MEI-217) in the male germline explains why its overall structure has been evolutionarily

200 maintained.

201

REC ATPase motifs are required for crossover formation

204 B motifs, suggesting that REC has ATP binding and hydrolysis activity (Kohl et al. 2012). It is

205 unknown whether the mei-MCM complex utilizes REC's putative ATPase activity for its

206 function in vivo. To test this, we used CRIPSR/Cas9 to introduce into rec mutations predicted to

207 disrupt Walker A and B motif functions (Figure 3A). The Walker A mutation (rec ${ }^{K A}$ ) results in 208 substitution of a conserved lysine residue with alanine; this mutation in other AAA+ ATPases, 209 including replicative MCMs, prevents binding of ATP (Bell and Botchan 2013). The Walker B 210 mutation $\left(\mathrm{rec}^{\mathrm{DA}}\right)$ results in substitution of a conserved aspartic acid with alanine; in MCMs and 211 other AAA+ ATPases, this mutation destroys the ability to coordinate $\mathrm{Mg}^{++}$for ATP hydrolysis 212 (Bochman et al. 2008).

213 We assayed crossover frequency along $2 \mathrm{~L}$ in $\operatorname{rec}^{K A}$ and $\operatorname{rec}^{D A}$ mutants (Figure 3B).

214 Surprisingly $\operatorname{rec}^{K A}$ ATP binding mutants exhibit a genetic length of $44.9 \mathrm{cM}$, which is not 215 significantly different from wild-type $(p=0.4016)$, suggesting that ATP binding by REC is not 216 required for crossover formation. Conversely, there is a severe reduction in crossovers in $\operatorname{rec}^{D A}$ 
mutants, with a genetic length of $1.6 \mathrm{cM}(p<0.0001)$, suggesting that REC's ability to hydrolyze ATP is required for crossover formation.

Because the genetic length of $\operatorname{rec}^{D A}$ is significantly lower than rec null mutants (Figure $3 \mathrm{~B}, p<0.0001)$, we hypothesized that $\operatorname{rec}^{D A}$ is an antimorphic mutation. To test this, we examined crossover levels and $X$ chromosome nondisjunction (NDJ) in $\mathrm{rec}^{D A} / \mathrm{rec}^{+}$(Figure 3B and $3 \mathrm{C}$, respectively). The genetic length of $2 \mathrm{~L}$ in $r e c^{D A /+}$ is slightly lower than wild-type, but not significantly different (43.9 $\mathrm{cM}$ and $45.8 \mathrm{cM}$, respectively; $p=0.35$ ). For $X$-NDJ, both wild-type and $\mathrm{rec}^{-} / \mathrm{rec}^{+}$mutants exhibit rates below $0.5 \%$, while $\mathrm{rec}^{\mathrm{DA}} / \mathrm{rec}^{+}$mutants exhibit a significant increase to $1.4 \%$ NDJ $(p<0.0001)$. These data support the conclusion that $\operatorname{rec}^{D A}$ is weakly antimorphic and suggest that $\operatorname{rec}^{D A}$ results in an inactive mei-MCM complex that is antagonistic to the wild-type complex. In light of these interpretations, we propose that the mei-MCM complex binds to recombination sites independent of REC binding to ATP, and that REC-dependent ATP hydrolysis is required for the removal of the mei-MCM complex from 230 these sites.

234 ATPase-dependent complexes. Rad51 paralogs, which form multi-protein complexes and contain 235 Walker A and B motifs, are proposed to exhibit ATPase activity in trans between adjacent 236 subunits, each of which contributes a Walker A or Walker B motif to the active site (Wu et al. 237 2004; Wu et al. 2005; Wiese et al. 2006). Because neither MEI-217 nor MEI-218 possess an 238 ATPase domain that harbors conserved key enzymatic residues (Figure 1B) (Kohl et al. 2012), 239 we propose that ATPase activity of the mei-MCM complex requires REC for ATP hydrolysis 
and an unknown mei-MCM protein for ATP binding. Alternatively, because REC is highly

241 diverged, its Walker A and B motifs may function non-canonically. Biochemical studies are

242 needed to test these hypotheses, but these may require identification of the putative missing

243 subunit.

REC-dependent ATP hydrolysis is required for MEI-9-dependent crossovers

To gain insight into the crossover pathways that are used in $\operatorname{rec}^{K A}$ and $\operatorname{rec}^{D A}$ mutants, we

247 examined whether these crossovers require the Class I endonuclease/resolvase. In Drosophila,

248 the catalytic subunit of the putative Class I meiosis-specific endonuclease is MEI-9 (Sekelsky et

249 al. 1995; Y1ldiz et al. 2002; Hatkevich et al. 2017). The 2L genetic length within a mei-9 mutant

250 is $2.75 \mathrm{cM}$ (Figure 4), demonstrating that at least $90 \%$ of crossovers are dependent upon MEI-9.

251 However, the genetic length in mei-9; rec mutants is not significantly different than that of rec

252 null single mutants (4.11 cM vs $4.66 \mathrm{cM}, p=0.64)$ suggesting that in the absence of REC, the

253 resulting crossovers are likely independent of MEI-9. Similarly, it has been shown previously

254 that mei-218 mei-9 double mutants do not have reduced crossovers compared to mei-218 single

255 mutants (Sekelsky et al. 1995), indicating that crossovers generated in the absence of the mei-

256 MCM complex are MEI-9-independent.

257 Because rec $^{K A}$ mutants exhibit the same distribution and number of crossovers as 258 wild-type (Figure 3B), we hypothesized that rec $^{K A}$ crossovers are dependent on MEI-9. To test 259 this, we examined genetic length across $2 \mathrm{~L}$ in $m e i-9 ;$ rec $^{K A}$ double mutants (Figure 4). Mutants 260 for mei-9; rec $^{K A}$ exhibit a genetic length of $2.72 \mathrm{cM}$, which is significantly decreased compared

261 to the rec $^{K A}$ single mutant $(p<0.0001)$, but not significantly different from mei-9 single mutants $262(p=0.94)$, showing that crossovers in $\operatorname{rec}^{K A}$ are indeed dependent upon MEI-9 nuclease. In 
263 contrast, we predicted that crossovers in $\operatorname{rec}^{D A}$ will be independent of MEI-9, similar to

264 crossovers generated in rec null mutants. We observe that mei-9; rec $^{D A}$ double mutants exhibit a

265 genetic length of $1.1 \mathrm{cM}$, which is significantly lower than that of mei-9 single mutants $(p<$

266 0.001). Importantly, crossing over in the mei-9; rec $^{D A}$ double mutant is not significantly different

267 than in $\operatorname{rec}^{D A}$ single mutants $(p=0.23)$, demonstrating that crossovers in rec $^{D A}$ are independent of

268 MEI-9 (Figure 4).

269 From these data we conclude that the crossovers in $\operatorname{rec}^{K A}$ mutants arise through the

270 normal, MEI-9-dependent pathway, whereas mitotic nucleases generate the residual crossovers

271 in rec $^{D A}$ mutants. These data show that $\mathrm{REC}^{\mathrm{KA}}$ functions normally in the Class I pathway, but

272 this pathway is nonfunctional in $r e c$ null and $r e c^{D A}$ mutants. We suggest that the REC's ability to

273 hydrolyze, but not bind, ATP is required for the formation of Class I crossovers.

REC ATPase motifs are required to prevent Class II crossovers

In wild-type Drosophila, most or all crossovers are generated through the Class I

277 pathway (Hatkevich et al. 2017), and these crossovers are dependent upon the mei-MCM

278 complex (Kohl et al. 2012). However, in Blm mutants, crossovers are generated exclusively

279 through the Class II pathway (Hatkevich et al. 2017). In Drosophila Blm mutants, meiotic

280 crossovers are decreased by 30\%, suggesting that the Class II pathway is less efficient at

281 generating crossovers than the Class I pathway, even though what may be the primary anti-

282 crossover protein, Blm helicase, is absent. It has previously been shown that loss of Blm

283 suppresses the high nondisjunction of mei-218 and rec mutants (Kohl et al. 2012). However, in

284 Blm rec double mutants, crossovers are increased significantly compared to Blm single mutants 
(Kohl et al. 2012), suggesting that REC and/or the mei-MCM complex has an anti-crossover role in Blm mutants, and therefore in the Class II crossover pathway.

To further understand the role of REC in the Class II pathway, we investigated whether REC's predicted ATP binding or hydrolysis function is required for its Class II anti-crossover rec double mutants. Conversely, if REC ATP binding or hydrolysis is not required, then double mutants will exhibit genetic lengths similar to that of Blm single mutants. significantly different than Blm rec mutants $(p=0.10)$ but significantly higher than Blm single mutants $\left(p<0.0001\right.$; Figure 5A). Similarly, $B \operatorname{Blm~rec}{ }^{D A}$ double mutants have a recombination rate of $53.4 \mathrm{cM}$, which not significantly different from Blm rec double mutants $(p=0.52)$, but 298 significantly higher than Blm single mutants $(p<0.0001)$. These results suggest that REC's predicted abilities to bind and hydrolyze ATP are both required for the inhibition of crossovers at

300 REC-associated Class II recombination sites. Therefore, it appears that REC forms different 301 complexes within the Class II pathway and Class I pathway. It is unknown whether this Class II 302 REC-associated complex requires the other mei-MCM proteins, and additional genetic studies 303 will be valuable to discern this.

In summary, the mei-MCMs are a family of diverged proteins that help to establish the 305 recombination landscape in Drosophila melanogaster by promoting Class I crossovers and 306 inhibiting Class II crossovers. Results obtained in this study have further elucidated meiotic 307 recombination roles for two mei-MCM proteins, MEI-218 and REC. While the N-terminus of 
308 MEI-218 is dispensable for crossover formation (Figure 2), REC's predicted ability to bind and

309 hydrolyze ATP exhibit differential requirements for regulating Class I and Class II crossover

310 formation. From our genetic analyses, we suggest that the Walker B motif of REC, but not the

311 Walker A motif, is required for promoting the formation Class I, MEI-9 dependent crossovers

312 (Figures 3 and 4). The weakly antimorphic phenotype of rec $^{D A}$ demonstrates that an impaired

313 REC Walker B mutant renders a poisonous complex - a complex in which we propose cannot be

314 released from recombination sites. Both Walker A and Walker B motifs block crossovers in the

315 Class II pathway, suggesting that REC forms different complexes to execute its pro- and anti-

316 crossover functions. Biochemical and cytological studies are needed to support or refute these

317 hypotheses.

\section{Acknowledgements}

319 We thank Juan Carvajal Garcia, Carolyn Turcotte, and anonymous reviewers for thoughtful

320 comments. This work was supported in part by a grant from the National Institute of General

321 Medical Sciences to J.S. under award 1R35 GM-118127. K.P.K. was supported in part by NIH

322 grant P20GM103499. T.H. was supported in part by NIH grants 5T32GM007092 and 323 1F31AG055157. 


\section{Figure Legends}

325 Figure 1. MCM protein structure and alignments. (A) Structural domains of Drosophila 326 melanogaster REC, MEI-217, MEI-218 and Mus musculus MCMDC2. Structural domains 327 identified using PHYRE 2 (Kohl et al. 2012). "MCM domain" corresponds to protein data bank 328 ID \#c2v16C and the AAA ATPase domains identified correspond to protein data bank ID 329 \#d1g8pa. The X on Dm MEI-218 and Mm MCMDC2 represents predicted inactive AAA ATPase 330 domains. (B) Consensus sequence for Walker A motif (Walker et al. 1982), and consensus 331 sequence for Walker B motif (Forsburg 2004). Identical or conserved amino acids are denoted 332 with black background. Arrows denote the conserved catalytic residues.

334 Schematic of transgenes for full length mei-218 and N-terminal deleted mei-218, in which the 335 first 526 amino acids are absent. (B) Map units of WT (Hatkevich et al. 2017), mei-218 (Kohl et 336 al. 2012), $m e i-218^{F L}$ and $m e i-218^{\Delta \mathrm{N}}$. Map units represented as centimorgans (cM). Error bars 337 indicate $95 \%$ confidence intervals. $n . s .=$ not significant $(p=0.61)$. (C) Crossover distribution 338 (solid lines) of $m e i-218^{F L}$ and $m e i-218^{\Delta \mathrm{N}}$ represented as $\mathrm{cM} / \mathrm{Mb} . \mathrm{Mb}$ is measured distance of 339 defined interval, excluding centromere, pericentromeric heterochromatin and transposable 340 elements. Dotted lines represent mean crossover density across 2L. Figure S5 details the cross 341 scheme of mei-218 transgene experiments. Refer to tables S1and S3 for complete data sets.

342 Figure 3. REC ATPase binding and hydrolysis requirements for crossover formation. (A)

343 Schematic representation of the mutated residues in $\operatorname{rec}^{K A}$ and $\operatorname{rec}^{D A}$. (B) Map units of WT 344 (Hatkevich et al. 2017), $\operatorname{rec}^{1} / r e c^{2}, \operatorname{rec}^{K A}$, and $r e c^{D A}$, rec ${ }^{D A} / \mathrm{rec}^{+}$. Map units represented as 345 centimorgans (cM). Error bars show 95\% confidence intervals. (C) Percent nondisjunction of 
$W T, \operatorname{rec}^{l} / \mathrm{rec}^{+}$, and $\mathrm{rec}^{\mathrm{DA}} / \mathrm{rec}^{+}$. (D) Model of possible complex depicting the functional Walker B $<0.0001$. Refer to tables S2 and S3 for complete data sets.

Figure 4. MEI-9-dependent crossovers in $\operatorname{rec}^{K A}$ and $\operatorname{rec}^{D A}$ mutants. Map units of $W T$

(Hatkevich et al. 2017), rec, mei-9, mei-9;rec, rec ${ }^{K A}$, mei-9;rec ${ }^{K A}, r e c^{D A}$, and mei-9;rec ${ }^{D A}$. Map

$p<0.001 * * * p<0.0001 ;\left(m e i-9\right.$ vs mei-9; $\left.\operatorname{rec}^{K A} p=0.94\right)\left(\operatorname{rec}^{D A}\right.$ vs $\left.m e i-9 ; \operatorname{rec}^{D A} p=0.23\right)$.

Refer to Table S3 for complete data set.

Figure 5. Requirements of REC ATPase activity in Blm function. Map units of WT

Blm rec ${ }^{D A}$. Map units represented as centimorgans (cM). Error bars show $95 \%$ confidence intervals. Refer to Table S3 for complete data set. *** $p<0.0001$. (Blm rec vs Blm rec ${ }^{K A} p=$

0.10) (Blm rec vs Blm $\left.\operatorname{rec}^{D A} p=0.52\right)$.

\section{References}

Baker, B. S., and A. T. C. Carpenter, 1972 Genetic analysis of sex chromosomal meiotic mutants in Drosophila melanogaster. Genetics 71: 255-286.

Beadle, G. W., 1932 A possible influence of the spindle fibre on crossing-over in Drosophila. Proc Natl Acad Sci U S A 18: 160-165.

Bell, S. D., and M. R. Botchan, 2013 The minichromosome maintenance replicative helicase. Cold Spring Harb Perspect Biol 5: a012807.

Blanton, H. L., S. J. Radford, S. Mcmahan, H. M. Kearney, J. G. Ibrahim et al., 2005 REC, Drosophila MCM8, drives formation of meiotic crossovers. PLoS Genet 1: e40.

Bochman, M. L., S. P. Bell and A. Schwacha, 2008 Subunit organization of Mcm2-7 and the unequal role of active sites in ATP hydrolysis and viability. Mol Cell Biol 28: 58655873. 
Brand, C. L., M. V. Cattani, S. B. Kingan, E. L. Landeen and D. C. Presgraves, 2018 Molecular evolution at a meiosis gene mediates species differences in the rate and patterning of recombination. Curr Biol 28: 1289-1295 e1284.

Collins, K. A., J. G. Callicoat, C. M. Lake, C. M. Mcclurken, K. P. Kohl et al., 2012 A germline clone screen on the $X$ chromosome reveals novel meiotic mutants in Drosophila melanogaster. G3 (Bethesda) 2: 1369-1377.

De Los Santos, T., N. Hunter, C. Lee, B. Larkin, J. Loidl et al., 2003 The Mus81/Mms4 endonuclease acts independently of double-Holliday junction resolution to promote a distinct subset of crossovers during meiosis in budding yeast. Genetics 164: 81-94.

Duffy, J. B., 2002 GAL4 system in Drosophila: a fly geneticist's Swiss army knife. Genesis 34: $1-15$.

Forsburg, S. L., 2004 Eukaryotic MCM proteins: beyond replication initiation. Microbiol Mol Biol Rev 68: 109-131.

Grell, R. F., 1978 Time of recombination in the Drosophila melanogaster oocyte: evidence from a temperature-sensitive recombination-deficient mutant. Proc Natl Acad Sci U S A 75: 3351-3354.

Hatkevich, T., K. P. Kohl, S. Mcmahan, M. A. Hartmann, A. M. Williams et al., 2017 Bloom syndrome helicase promotes meiotic crossover patterning and homolog disjunction. Curr Biol 27: 96-102.

Hatkevich, T., and J. Sekelsky, 2017 Bloom syndrome helicase in meiosis: Pro-crossover functions of an anti-crossover protein. Bioessays 39.

Hollingsworth, N. M., and S. J. Brill, 2004 The Mus81 solution to resolution: generating meiotic crossovers without Holliday junctions. Genes Dev 18: 117-125.

Iyer, L. M., D. D. Leipe, E. V. Koonin and L. Aravind, 2004 Evolutionary history and higher order classification of AAA+ ATPases. J Struct Biol 146: 11-31.

Kohl, K. P., C. D. Jones and J. Sekelsky, 2012 Evolution of an MCM complex in flies that promotes meiotic crossovers by blocking BLM helicase. Science 338: 1363-1365.

Lake, C. M., and R. S. Hawley, 2012 The molecular control of meiotic chromosomal behavior: events in early meiotic prophase in Drosophila oocytes. Annu Rev Physiol 74: 425-451.

Liu, H., J. K. Jang, J. Graham, K. Nycz and K. S. Mckim, 2000 Two genes required for meiotic recombination in Drosophila are expressed from a dicistronic message. Genetics 154: 1735-1746.

Matsubayashi, H., and M. T. Yamamoto, 2003 REC, a new member of the MCM-related protein family, is required for meiotic recombination in Drosophila. Genes Genet Syst 78: 363371.

Mckim, K. S., J. B. Dahmus and R. S. Hawley, 1996 Cloning of the Drosophila melanogaster meiotic recombination gene mei-218: a genetic and molecular analysis of interval 15E. Genetics 144: 215-228.

Mcvey, M., S. L. Andersen, Y. Broze and J. Sekelsky, 2007 Multiple functions of Drosophila BLM helicase in maintenance of genome stability. Genetics 176: 1979-1992.

Miller, D. E., C. B. Smith, N. Yeganeh Kazemi, A. J. Cockrell, A. V. Arvanitakis et al., 2016 Whole-genome analysis of individual meiotic events in Drosophila melanogaster reveals that noncrossover gene conversions are insensitive to interference and the centromere effect. Genetics 203: 159-171. 
Sekelsky, J. J., K. S. Mckim, G. M. Chin and R. S. Hawley, 1995 The Drosophila meiotic recombination gene mei-9 encodes a homologue of the yeast excision repair protein Rad1. Genetics 141: 619-627.

Thurmond, J., J. L. Goodman, V. B. Strelets, H. Attrill, L. S. Gramates et al., 2018 FlyBase 2.0: The next Generation. Nucleic Acids Res 47: D759-765.

Walker, J. E., M. Saraste, M. J. Runswick and N. J. Gay, 1982 Distantly related sequences in the alpha- and beta-subunits of ATP synthase, myosin, kinases and other ATP-requiring enzymes and a common nucleotide binding fold. EMBO J 1: 945-951.

Wiese, C., J. M. Hinz, R. S. Tebbs, P. B. Nham, S. S. Urbin et al., 2006 Disparate requirements for the Walker A and B ATPase motifs of human RAD51D in homologous recombination. Nucleic Acids Res 34: 2833-2843.

Wu, Y., Y. He, I. A. Moya, X. Qian and Y. Luo, 2004 Crystal structure of archaeal recombinase RADA: a snapshot of its extended conformation. Mol Cell 15: 423-435.

Wu, Y., X. Qian, Y. He, I. A. Moya and Y. Luo, 2005 Crystal structure of an ATPase-active form of Rad51 homolog from Methanococcus voltae. Insights into potassium dependence. J Biol Chem 280: 722-728.

Yıld1z, Ö., H. Kearney, B. C. Kramer and J. J. Sekelsky, 2004 Mutational analysis of the Drosophila DNA repair and recombination gene mei-9. Genetics 167: 263-273.

Yıld1z, Ö., S. Majumder, B. C. Kramer and J. Sekelsky, 2002 Drosophila MUS312 interacts with the nucleotide excision repair endonuclease MEI-9 to generate meiotic crossovers. Mol Cell 10: 1503-1509.

\section{Author Summary}

Crossover formation between homologs is essential for accurate segregation at the end of meiosis I. Crossovers are typically formed through two pathways: Class I and Class II. The meiMCMs are a class of proteins that promote Class I crossover formation and prohibit Class II crossovers in Drosophila. Although the mei-MCMs are conserved, little is known about their function. Here, we investigate the roles of two mei-MCMs, REC and MEI-218, in Class I and Class II crossover formation in Drosophila. From results in this study, we generate novel, testable hypotheses to further elucidate the meiotic function of the mei-MCM proteins. 
A Dm REC

500 800850

Mm MCMDC2

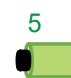

$275 \quad 316$

618

681

B

$D m \underline{M E I}-218$ Mrn MCMDC2

Dm MCM

Mrn MCNБ

$D m$ RE C

Mm MCNB
Walker A

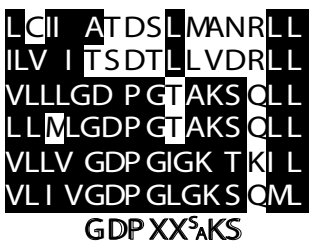

GDP XX ${ }^{S}{ }_{A K S}$

$\uparrow$
Walker B

AS- PLL L AQdGVYYAGDVNRL

OAGSALL AKGGI CFI GDLTS H

EGGAMV ADGGVVCID E FDKM

EGGAMV ADGGVVCID E FDKM

AGSLMNASGGGHCTLDDVDKL

EAGALVL GDOGI CGID E FDKM

『DEFDKM

$\uparrow$ 
A

$m e i-218^{F L}$

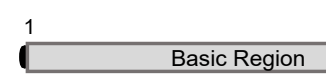

500 Acidic Region 800

1116 $m e i-218^{\Delta N}$

527

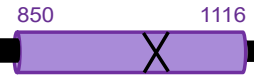

1186

B

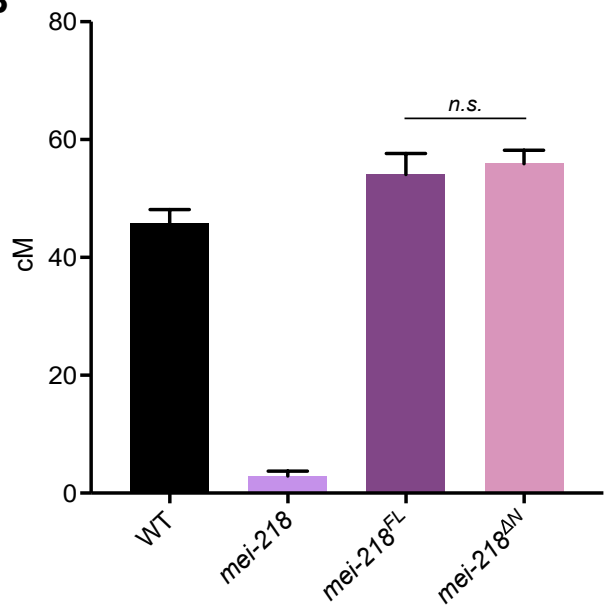

C

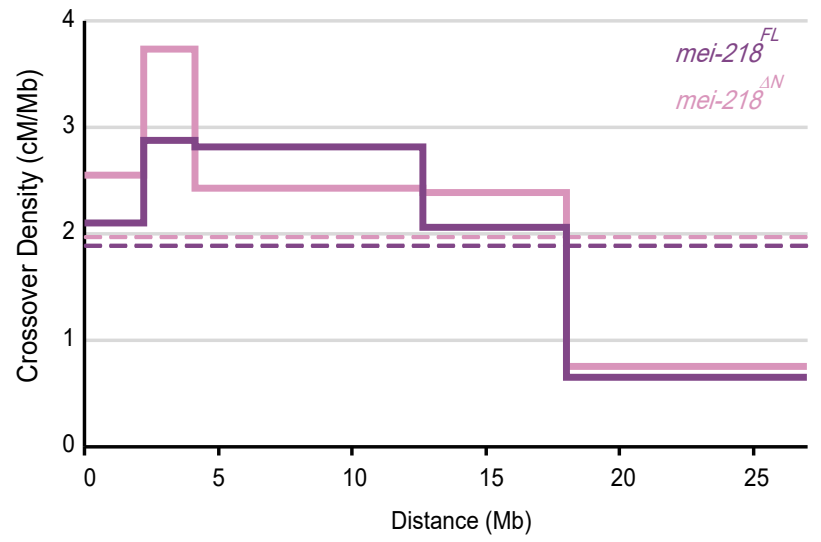


A
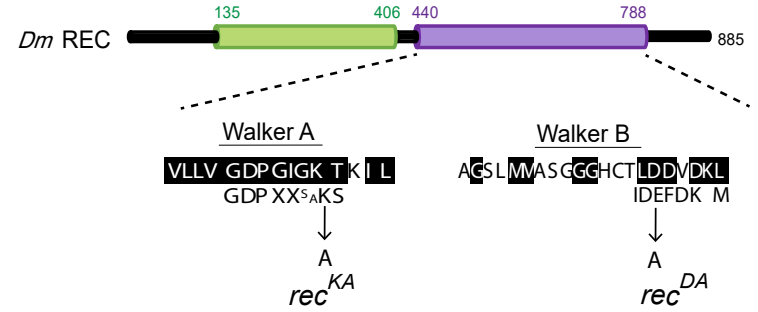

B

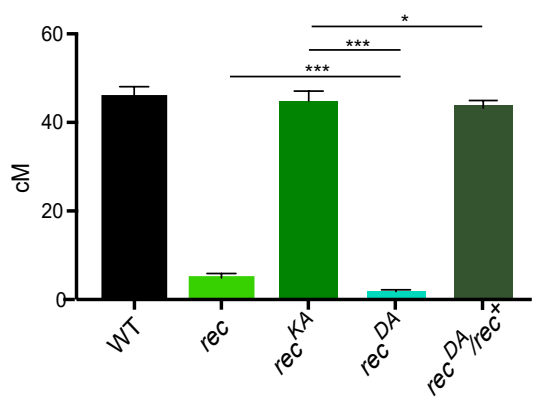

C

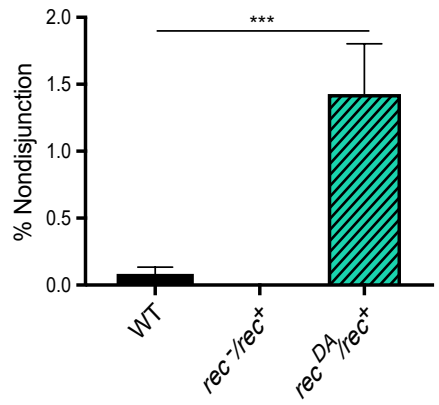




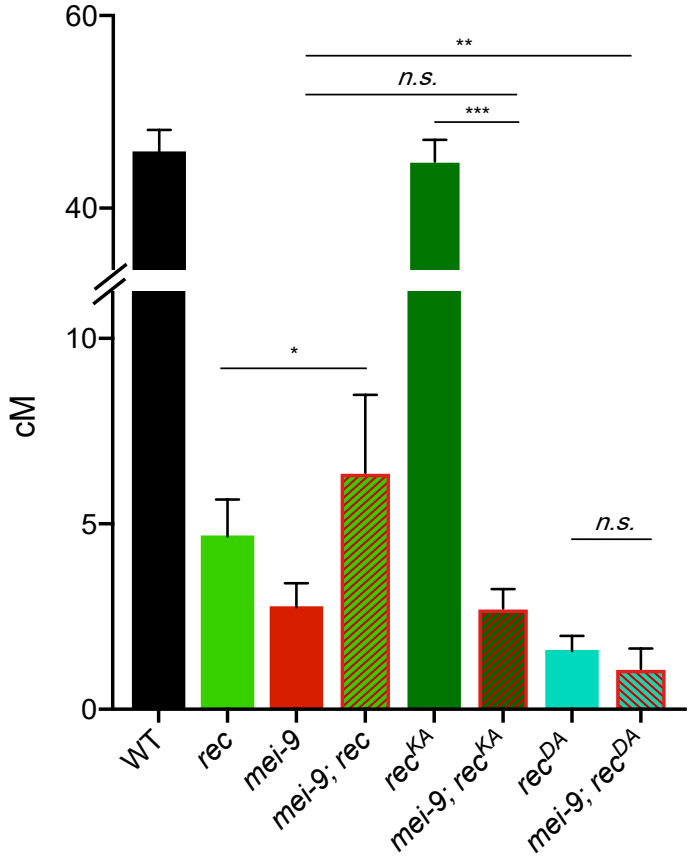




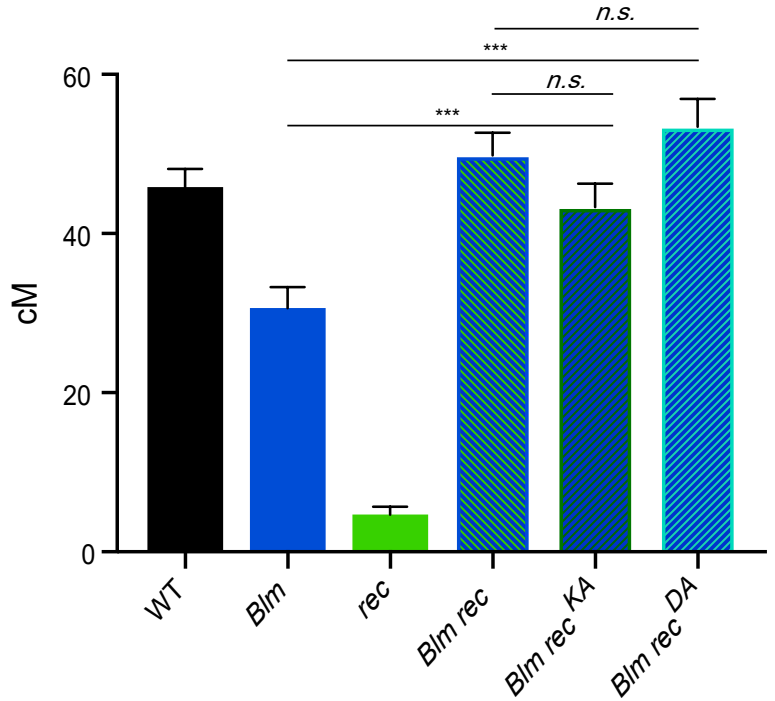

